\title{
Depth dependence of nanohardness in a CuAlNi single crystal shape memory alloy
}

\author{
Cheng Liu ${ }^{\text {a,b }}$, Qingping Sun ${ }^{\text {a.* }}$, Yapu Zhao ${ }^{\text {b }}$, Tongxi Yua ${ }^{\text {a }}$ \\ ${ }^{a}$ Department of Mechanical Engineering, Hong Kong University of Science and Technology, \\ Clear Water Bay, Kowloon, Hong Kong, SAR, China \\ ${ }^{b}$ State Key Laboratory of Nonlinear Mechanics, Institute of Mechanics, Chinese Academy of \\ Sciences, 15 Zhongguancun Road, Beijing 100080, China
}

\begin{abstract}
Instrumented nanoindentation was employed to study the depth dependence of nanohardness in a CuAlNi single crystal shape memory alloy that exhibits shape memory effect (SME). A Berkovich indenter and a cube corner indenter were used in this study, and the applied maximum indentation loads ranged from $10000 \mu \mathrm{N}$ to $200 \mu \mathrm{N}$. Indentation of the austenite (A) phase of CuAlNi underwent a stress-induced phase transition. The measured nanohardness of $\mathrm{A}$ and $\mathrm{M}$ phases depends on both the indentation depth and the indenter gcometry.
\end{abstract}

Keywords: Nanoindentation, Phase transition, CuAlNi single crystal shape memory alloy

\section{Introduction}

The shape memory effect (SME) and superelasticity (SE) occur in shape memory alloys (SMAs). The underlying mechanism of these properties is the stress-induced martensitic transformation. Many experimental and numerical studies of this mechanism have been focused on: (1) the overall response of SMAs under different temperatures, stress states and orientations; (2) the morphology of martensite and the crystallography; and (3) the micromacro-related deformation behavior. SMAs have been exploited for many engineering In recent years, they are used in micro-electromechanical-systems (MEMS) and novel medical devices [1]. For MEMS, NiTi and $\mathrm{NiTiCu}$ SMAs thin films have been fabricated and used

* Corresponding author. Tel. : +852-23588655; fax: +852 23581543

Email address: meqpsun@ust.hk in micro-actuators and fluid flow valves. In the medical field, NiTi superelastic micro-tubes and wires are successfully used for artery stents and surgical tools. Consequently, the deformation and properties of SMAs in small scales have attracted great interest on the study of SMAs. Sun et al.[2] studied the size effect on the macroscopic martensite band nucleation stress of $\mathrm{NiTi}$ thin wires under tension. They found that the smaller the diameter of the wire the higher the nucleation stress. Gall et al.[3] used transmission electron microspcopy (TEM) to study the deformation machanisms for the indented NiTi SMAs. They found that indentation of solutionized NiTi SMAs could induce inelastic deformation via dislocation activity and a stress-induced martensitic transformation.

To study the mechanical properties of materials in a small scale or the size effect, one of the useful methods is the instrumented nanoindentation. In ample experiments the hardness of metal is observed to increase with 
'decreasing indentation load. However, a little attention had been paid to the study of the depth dependence of hardness in the SMAs that are capable of undergoing a stress-induced martensitic transformation during indentation. In our study, nanohardness variations with indentation load were investigated in a CuAlNi single crystal shape memory alloy.

\section{Material and equipment}

\subsection{Material}

A CuAINi single crystal specimen was studied in the present test. The transition temperatures are $M_{s}=279 \mathrm{~K}, M_{f}=276 K, A_{s}=$ $308 \mathrm{~K}$ and $A_{f}=318 \mathrm{~K}$. At the room temperature, the specimen exhibits SME. Austenite (A) and martensite (M) phases can coexist in the specimen by either loading-unloading or first heating above $A_{f}$ and then cooling to the temperature above $\mathrm{M}_{\mathrm{f}}$. The specimen size was $8 \times 3.5 \times 2 \mathrm{~mm}$.

\subsection{Equipment}

Hysitron Tribolndenter was used to conduct the nanoindentation tests. From the loadingunloading curve, the nanohardness and the elastic modulus of materials were estimated using the method proposed by Oliver and Pharr [4]. Two kinds of indenter tips were used in this study. One was a diamond Berkovich tip with a face angle of $142.3^{\circ}$ and the other was a diamond cube corner tip with a face angle of $90^{\circ}$. The applied maximum indentation loads ranged from $10000 \mu \mathrm{N}$ to $200 \mu \mathrm{N}$. The indentation tests were performed in both $\mathrm{A}$ and $\mathrm{M}$ zones of CuAINi at room temperature. For each zone, indentation at each load was repeated three times.

\section{Results and discussion}

\subsection{AFM images of $A$ and $M$ phases}

The morphology of CuAINi alloy was observed by atomic force microscope (AFM) before indentation. Figure 1 is a 3D image of the specimen surface. An obvious sharp A-M interface can be seen in the figure. On the left side of the interface is the $M$ phase inside in which small shallow bands (martensite twins) can be seen. On the right side is the A phase. The width of the martensite twin is about $7.7 \mu \mathrm{m}$.

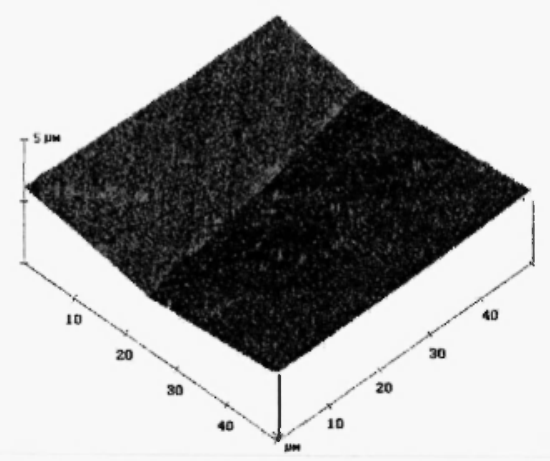

Fig. I A 3D AFM image of the specimen surface. (left: twinned $M$ phase; right: A phase)

\subsection{Nanohardness and reduced elastic moduli of $A$ and $M$ phases}

Figure 2(a) shows the typical loadingunloading curves by the Berkovich tip indented on $A$ and $M$ phases respectively. It can be seen that for different applied maximum loads the loading curves of A phase coincide one another and the unlaoding curves are alomost parallel. The same phenomena can be observed for the $M$ phase. Comparing the set of curves of A phase with that of $\mathrm{M}$ phase, it is found that the depth in the A phase is less than that in the $M$ phase at the same indentation load. Furthermore, all the unloading curves of $\mathrm{A}$ and $\mathrm{M}$ phases are nearly parallel although the loading curves of $A$ and $M$ phases are different. The same phenomena can also be found in the CuAlNi alloy indented by the cube corner tip (Fig. 2(b)). The curves revealed that inelastic deformation occurred during indentation. There are two possible origins to the inelastic deformation. One is the stress-induced $A \rightarrow M$ transition for the A phase or the stress-induced martensite-reorientation in the $M$ phase. The other is the dislocation activity in both $\mathrm{A}$ and $\mathrm{M}$ phases.

Figure 3 (a) shows the depth dependence of nanohardness in both $A$ and $M$ phases using the Berkovich indenter. It is found that the nanohardness increases in both phases with 
decreasing indentation depth (or load). Moreover, the nanohardness obtained in the A phase is higher than that in the $M$ phase at the same depth. It is known that the hardness is a measure of the material's yield strength. The obtained data implies that the stress to induce martensitereorientation in the $M$ phase is lower than that to induce martensitic transformation in the A phase.

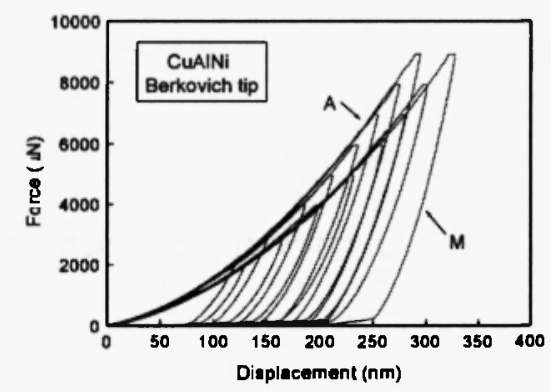

(a)

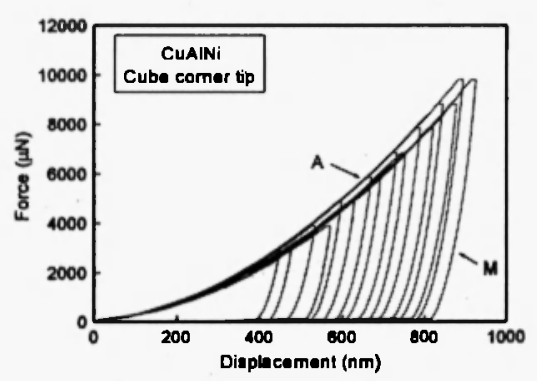

(b)

Fig. 2 Force-displacement plots for the CuAlNi alloy indented (a) by the Berkovich tip;

(b) by the cube corner tip.

Figure 3 (b) shows the depth dependence of nanohardness of $A$ and $M$ phases using the cube corner indenter. Compared with the results obtained by the Berkovich indenter, the cube corner indenter, with a sharper tip, penetrated much deeper in both $\mathrm{A}$ and $\mathrm{M}$ phases. Under the indentation load $10000 \mu \mathrm{N}$, the penetration depth was up to approximately $800 \mathrm{~nm}$ under the cube corner tip, while the depth was only $300 \mathrm{~nm}$ for the Berkovich tip. Also, the nanohardness increased with decreasing depth in both $\mathrm{A}$ and $\mathrm{M}$ phases using the cube corner tip, and the nanohardness was slightly higher in the A phase than in the $M$ phase. Comparing Fig. 3(a) with Fig. 3(b), it is found that at the same depth the nanohardness obtained by the cube corner tip is higher than that by the Berkovich tip. This may be explained by the strain gradient effect: the strain gradient is higher around the cube corner tip than that around the Berkovich tip.

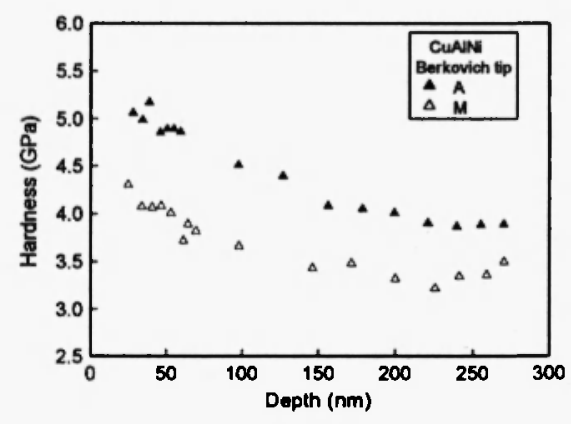

(a)

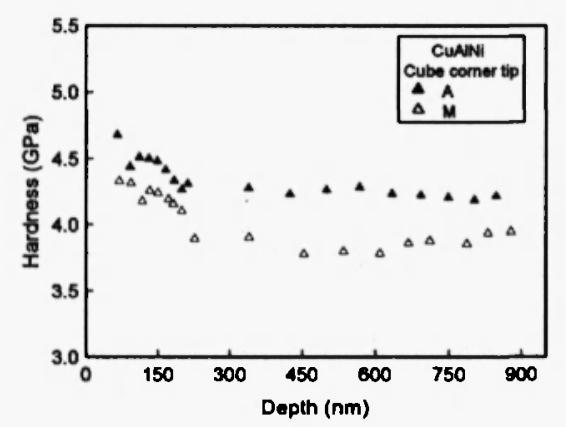

(b)

Fig. 3 Depth dependence of nanohardness of $A$ and $M$ phases using (a) the Berkovich tip, (b) the cube corner tip.

(The data is the average value at each load)

Figure 4 is the reduced elastic moduli in A and $M$ zones under different indentation depth tested by the Berkovich tip. It is found that the

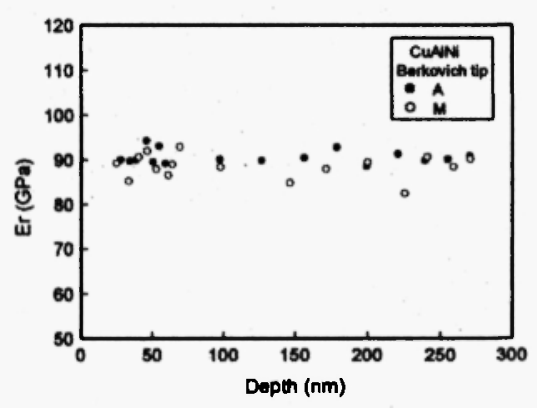

Fig. 4 Reduced elastic moduli in $\mathrm{A}$ and $\mathrm{M}$ zones using the Berkovich tip.

(The data is the average value at each load) 
reduced elastic moduli in $\mathrm{A}$ and $\mathrm{M}$ zones are nearly equal. The same phenomenon can also be found in the CuAlNi alloy indented by the cube corner tip (not shown here). From the parallel unloading curves of $A$ and $M$ phases in Figs. 2(a) and (b), it is inferred that indentation is not suitable for the measurement of the elastic modulus of $\mathrm{A}$ phase that undergoes a stressinduced transformation during indentation.

\section{Conclusions}

1) The force-displacement curves exhibit that inelastic deformation occurred in both $\mathrm{A}$ and $M$ phases. There are two possible contributions to the inelastic deformation. One is the stress-induced $\mathrm{A} \rightarrow \mathrm{M}$ transition for the $A$ phase or the stress-induced martensite-reorientation in the $\mathrm{M}$ phase. The other is the dislocation activity in both $\mathrm{A}$ and $M$ phases.

2) For a given-shaped indenter, all the unloading curves of $A$ and $M$ phases are almost parallel although the loading curves of $A$ and $M$ phases are different. It is inferred that indentation is not suitable for the measurement of the elastic modulus of $A$ phase that undergoes a stress-induced martensitic transformation during indentation.

3) The nanohardness of $A$ and $M$ phases depends on the indentation depth and the indenter geometry. For a given-shaped indenter, the nanohardness increases in both $A$ and $M$ phases with decreasing indentation depth, and the nanohardness obtained in the A phase is higher than that in the $M$ phase. For the same indentation depth, the nanohardness obtained by the cube corner tip in $A$ and $M$ phases is higher than that by the Berkovich tip.

\section{Acknowledgments}

The research project is supported by the Joint Lab on Microsystems between the Hong Kong University of Science and Technology (HKUST) and the Institute of Mechanics, CAS,
China, under a grant from HKUST (Project No. CMI00/01.EG02). The authors gratefully acknowledge Mr. William Chi Chung Wong and Mr. Peter Ka Chun Lee of HKUST for their sincere helps in nanoindentation work. We also thank Dr. Jie Xhie and Mr. Jiaqi Zheng of HKUST for the assistance in the AFM work and the specimen preparation.

\section{References}

[1] Ostuka K and Kakeshita T, Science and technology of shape-memory alloys: new developments, MRS Bulletin, February 2002, 91-100.

[2] Sun Q P, Li Z Q and Tse K K, Proceeding of IUTAM Symposium on smart structures and structronic systemm Magdeburgm Germany, Sept. 26-29,2000, Kluwer Academic Publishers.

[3] Gall K, Juntunen K, Marer H J, Sehitoglu H and Chumlyakov Y I, Instrumented microindentation of NiTi shape memory alloys, Acta mater. 49(2001) 3205-3217.

[4] Oliver W C and Pharr G M, An improved technique for determining hardness and elastic modulus using load and displacement sensing indentation experiments, J. Mater. Res. 7(6) (1992) 1564-1583. 